\title{
CONOCER, ENSEÑAR Y EDUCAR TIENEN DISTINTO SIGNIFICADO, LA DIFERENCIA PERMITE HABLAR CON SENTIDO DE ENSEÑANZA EDUCATIVA. UNA MIRADA MESOAXIOLÓGICA
}

\section{KNOWING, TEACHING AND EDUCATING HAVE A DIFFERENT MEANING, THE DIFFERENCE ALLOWS TALKING WITH A SENSE OF EDUCATIVE TEACHING. A MESOAXIOLOGICA LOOK}

\section{José Manuel TOURIÑÁN LÓPEZ}

Catedrático de Teoría de la Educación, Universidad de Santiago de Compostela Facultad de Ciencias de la Educación. Departamento de Pedagogía y Didáctica. Campus Vida s/n. 15782. Santiago de Compostela. 
1 José Manuel Touriñán López

Premio Internacional Educa-Redipe 2019 (Trayectoria profesional). Resolución de 18 de mayo de 2019

Maestro de Enseñanza Primaria (1969). Licenciado (1974) y Doctor (1978) en Pedagogía con premio extraordinario en ambos casos. Catedrático de Universidad (1988). Evaluador-auditor de programas universitarios, proyectos de investigación y publicaciones científicas en organismos oficiales y revistas profesionales. Autor de más de 250 estudios (en forma de artículos de revistas de investigación y capítulos de libros especializados), 40 libros escritos como autor único y 10 más en coautoría. Ha recibido premios autonómicos, nacionales e internacionales. Su trayectoria profesional ha sido biografiada en repertorios europeos y americanos. Es coordinador de la red RIPEME (Red internacional de pedagogía mesoaxiológica), integrada en la Red iberoamericana de pedagogía (Redipe), y del grupo de investigación Tercera Generación (TeXe), de la Universidad de Santiago de Compostela en la que imparte docencia de Teoría de la Educación, Política de la Educación y Función Pedagógica.

Sus líneas de investigación sobre desarrollo de sistemas educativos, intervención pedagógica y función pedagógica convergen actualmente en la línea Pedagogía mesoaxiológica y construcción de ámbitos de educación: valoración del contenido de las áreas de experiencia cultural como educación y fundamentación del conocimiento necesario para la construcción ámbitos de educación, de manera que se integren en el diseño educativo valores comunes de educación y valores educativos específicos de cada área cultural (Proyecto PIIR 004 Educere Area. Educar con las áreas culturales), disponible en la dirección http:// dondestalaeducacion.com/files/5615/6485/0752/Proy Educere Area-Lin Invest PMyCAE.pdf

Por Resolución de 18 de mayo de 2019, se le ha otorgado el Premio Educa-Redipe 2019 (Trayectoria profesional).

Webs Particulares: $h$ ttp://dondestalaeducacion.com/ https:// www.youtube.com/channel/UCzbNAzwFApNMViix5_HDzOW Researcher ID: $\quad$ http://www.researcherid.com/ rid/L-1032-2014 Orcid: http://orcid.org/0000-0002-7553$\underline{4483}$

\section{RESUMEN}

Conocer, enseñar y educar no significan lo mismo. Para enseñar, además de conocer, hemos de conseguir que el educando haga cosas para que llegue a conocer y actuar. Para educar hemos de dar un paso más allá, porque hay enseñanzas que no educan: tenemos que valorar cada medio que utilizamos como educativo y ajustarlo a los rasgos inherentes al significado de educar. El objetivo de conocer es llegar a saber, el objetivo de enseñar es hacer saber y actuar a otro. El objetivo de educar es conseguir que el educando se capacite para decidir y realizar sus proyectos y se convierta en agente actor y autor de estos.
Este artículo pretende la formación de criterio sobre el significado de 'educación' y la importancia de la Pedagogía en la construcción de ámbitos de educación. El conocimiento de la educación hace posible la construcción de ámbitos de educación con las áreas culturales. El significado de educación es la conjunción de carácter y sentido pedagógicos. El carácter determina el significado, el sentido cualifica el significado.

\section{ABSTRACT}

Knowing, teaching and educating do not mean the same thing. To teach, in addition to knowing, we have to get the student to do things so that he gets to know and act. To educate we have to go one step further, because there are teachings that do not educate: we have to value each medium that we use as educational and adjust it to the characteristics inherent in the meaning of educating. The objective of knowing is to get to know, the objective of teaching is to make that the educatee knows and acts. The objective of educating is to get the educatee to be able to decide and carry out their projects and become an agent, actor and author, of those projects.

This article looks for the formation of criteria about the meaning of "education" and the importance of Pedagogy for the construction of educational fields. Knowledge of education makes it possible the creation of educational fields by means of cultural areas. The meaning of education is the conjunction of pedagogical character and sense. The character determines the meaning, the sense gives it quality.

\section{PALABRAS CLAVE}

Concepto de educación, Conocimiento de educación, Ámbito de educación, Significado de educar, Pedagogía mesoaxiológica.

\section{KEY WORDS}

Concept of Education, Knowledge of Education, 
Field of Education, Meaning of Education, Mesoaxiological Pedagogy.

\section{CONTENIDO}

La expresión "Enseñar educando" sería una redundancia, si enseñar y educar significasen lo mismo. Esa expresión 'Enseñar educando', para que tenga sentido gramatical, quiere decir necesariamente que enseñar y educar no significan lo mismo, que educar cualifica el significado de enseñar $y$, por consiguiente, no toda enseñanza es educación, pues hay enseñanzas que no educan.

En Pedagogía mesoaxiológica, conocer, enseñar y educar no significan lo mismo; el conocimiento de la educación determina el concepto de ámbito de educación sobre el conocimiento de áreas culturales y tiene sentido afirmar que enseñar educando expresa un grado singular de compromiso y orientación formativa para la acción instructiva y el contenido instructivo².

Desde el punto de vista antropológico, la educación es cultura y, por tanto, tiene sentido afirmar que la función del profesional de la educación es transmitir cultura. Pero, si además afirmamos que los términos educacionales carecen de contenido propio, los conocimientos de las diversas áreas culturales se convierten en el eje de toda actividad pedagógica hasta el extremo de que los mismos profesionales de la educación tendrían que llegar a aceptar, por coherencia, que su formación es simplemente el conocimiento de esas áreas culturales y que conocer, enseñar y educar serían la misma cosa. Para mí, por principio de significado, conocer un área cultural no es enseñar, porque el conocimiento puede estar separado de la acción y enseñar no es educar, porque podemos afirmar que hay enseñanzas que no educan, con fundamento en el significado propio de esos

2 Touriñán, J. M. (2017). Mentalidad pedagógica y diseño educativo. De la pedagogía general a las pedagogías aplicadas en la función de educar. Santiago de Compostela: Andavira. términos. La lógica de saber no es la lógica de hacer saber y esta no es la lógica de educar, necesariamente ${ }^{3}$.

El nivel de las investigaciones pedagógicas actuales permite afirmar que hay razones suficientes para distinguir y no confundir en el lenguaje técnico el conocimiento de la educación y los conocimientos de las áreas culturales. Hoy, desde la perspectiva de la Pedagogía y en relación con las áreas culturales, es verdad que el conocimiento del área cultural es un componente de la acción educativa, pero el conocimiento del área cultural tiene un protagonismo distinto cuando hablamos de "conocer un área cultural", "enseñar un área cultural" y "educar con un área cultural". Esto que decimos, es obvio, si pensamos en un caso concreto, pues no es lo mismo "conocer Historia", que "enseñar Historia" que "educar con la Historia", y así sucesivamente con cada área de experiencia que se constituye en objeto de enseñanza y ámbito de educación en un proceso educativo.

Desde el punto de vista del conocimiento de la educación, al que enseña se le requiere un determinado nivel de formación relativo al conocimiento del área que será objeto de la enseñanza (área de experiencia y formas de expresión adecuadas al área), pero de ahí no se sigue que enseñar un área sea conocer ese área y que educar sea simplemente enseñar el área. Es innegable, dado el actual desarrollo del conocimiento de la educación, que todos los profesores no requieren el mismo nivel de pericia en el área cultural de experiencia que enseñan, según cuál sea su nivel de ubicación en el sistema educativo, y que todos los profesores no deben tener el mismo conocimiento pedagógico, dependiendo de cuál sea el nivel del sistema educativo en el que se trabaje. No hay que saber

3 Touriñán, J. M. (2018a). Concepto de educación y conocimiento de la educación. The Concept of Education and the Knowledge of Education. Colombia-Nueva York: Redipe (Bowker-Books). 
la misma Historia para ser profesor de Historia en el nivel de educación primaria, secundaria, o terciaria.

Conocer, en el amplio sentido de rendimiento identificado con las expresiones "sé qué, sé cómo y sé hacer", no se confunde con enseñar. Aptitudes y competencias para conocer y aptitudes y competencias para enseñar no se subsumen unas en las otras, ni tampoco ambas vacían de significado a la expresión "educar con" un área cultural. El análisis detenido del contexto pedagógico da pie para sostener que el conocimiento de las áreas culturales no es el conocimiento de la educación y que tiene sentido distinguir conocer, enseñar y educar, porque ${ }^{4}$ :

a) Si bien es verdad que una buena parte de los objetivos de la educación tiene algo que ver con los contenidos de las áreas culturales en la enseñanza, el ámbito de los objetivos no se agota en los ámbitos de las áreas culturales, ni siquiera en la docencia. La función pedagógica, referida a la docencia, no se agota en saber la información cultural correspondiente a un tema de un área cultural en una clase; antes bien, la función pedagógica se pone de manifiesto cuando se sabe qué tipos de destrezas, hábitos, actitudes, etc., de los diversos dominios que señalan las taxonomías, se están potenciando al trabajar de manera especial en ese tema. La cuestión, en la docencia, no es saber tanto sobre un área como el especialista, sino saber qué objetivos de conocimiento se logran y cómo se logran al enseñar un tema del área y qué destrezas, hábitos, actitudes, conocimientos y competencias estamos desarrollando al enseñar ese tema.

b) La identificación del conocimiento de las áreas culturales con el conocimiento

4 Touriñán, J. M. (2014). Dónde está la educación. Actividad común interna y elementos estructurales de la intervención. A Coruña: Netbiblo (http://dondestalaeducacion. com). de la educación fomenta una situación pedagógica insostenible: la tendencia a evaluar el rendimiento escolar fundamentalmente por los niveles de información cultural de área. Sin que ello signifique que cualquier contenido sea puramente formal y sirva para alcanzar cualquier tipo de destreza, es posible afirmar que, aunque no con el mismo nivel de eficacia, desde el punto de vista pedagógico, con uno sólo de los temas culturales del programa que debe estudiar un alumno de secundaria, por ejemplo, se podrían poner en marcha las estrategias pedagógicas conducentes al logro de casi todos los objetivos educativos del programa, a excepción de la información cultural específica del área.

c) Incluso identificando conocimiento de la educación y conocimiento de áreas culturales, se puede entender que hay un determinado conocimiento de la educación, hablando de la enseñanza, que no es el conocimiento de las áreas culturales: el conocimiento de la transmisión de los conocimientos de esas áreas culturales. La educación tendría efectivamente como misión, por ejemplo, la transmisión de conocimiento acerca del arte y lo artístico. En este caso, que ese conocimiento sea fiable y válido es problema de los historiadores del arte, de los críticos del arte y de los investigadores de esa área cultural; el conocimiento de la educación para la enseñanza sería, en este caso, el conocimiento de las estrategias de intervención.

d) Atendiendo a lo anterior, es obvio que existe una competencia distinta para educar y enseñar que para conocer un área cultural específica. En efecto, los conocimientos teóricos, tecnológicos y práxicos que se constituyen en objetivos 
de instrucción en la enseñanza, no los crea el profesional de la educación; son los investigadores de cada área cultural los que los crean. Al profesional de la educación le corresponde, con fundamento de elección técnica, decidir: si el educando puede aprenderlos; si son coherentes con la representación conceptual de la intervención educativa; si tienen fundamento teórico, tecnológico y práxico, según el caso, en el conocimiento de la educación para ser utilizados como instrumento de la educación; qué nivel de contenidos es adecuado en un caso concreto, cual es el método de enseñanza adecuado y qué destrezas, hábitos y actitudes, conocimientos y competencias educativas se pueden desarrollar con la enseñanza de ese conocimiento. Es decir, el profesional de la educación domina los conocimientos teóricos, tecnológicos y práxicos del área cultural que va a enseñar, al nivel suficiente para enseñarlos; pero, como profesional de la educación, domina el conocimiento de la educación que le permite justificar y explicar la conversión de esos conocimientos de un área cultural en objetivo o instrumento de la intervención pedagógica.

e) Desde el punto de vista de la competencia educativa, la clave del conocimiento que es válido para educar no está en el dominio de las áreas culturales, como si fuera el especialista de ese área cultural (artista, historiador, químico, u otros), sino en el dominio de la competencia pedagógica que le capacita para ver y utilizar el contenido cultural como instrumento y meta de acción educativa en un caso concreto, de manera tal que ese contenido cultural sea utilizado como instrumento para desarrollar en cada educando el carácter y sentido propios del significado de 'educación'. El conocimiento de la educación capacita al profesional de la educación, por ejemplo, no sólo para establecer el valor educativo de un contenido cultural y participar en el proceso de decidir su conversión en fin o meta de un determinado nivel educativo, sino también para establecer programas de intervención ajustados a hechos y decisiones pedagógicas que hagan efectiva la meta propuesta.

Hablar de conocimiento de la educación no implica interrogarse directamente acerca de los saberes de las áreas culturales. Cuando hablamos de "el conocimiento de la educación", es más apropiado preguntarse por qué determinados conocimientos se constituyen en meta o instrumento de la acción educativa o por qué es educable la dimensión cognitiva del hombre. $Y$ así como de los conocimientos de cada área cultural podrían hablarnos, según el caso y con propiedad, el historiador, el geógrafo, el matemático, el físico, el crítico de arte, etc., porque son especialistas en cada una de esas áreas culturales, no cabe duda que responder adecuadamente a, si tal o cual contenido histórico, matemático, físico, artístico, etcétera, debe constituirse en el contenido de la acción educativa que realizamos con un determinado sujeto, o a cómo cultivar su sentido crítico, exige interrogarse acerca de la educación como objeto de conocimiento.

En el primer supuesto, los conocimientos -la historia, la matemática, la física, etc.- son el objeto científico de estudio; en los dos casos del segundo supuesto, la transmisión misma y la mejora de la capacidad de conocer se convierten en objeto específico de la reflexión científica en forma de Didáctica y de Pedagogía cognitiva, según el caso. $\mathrm{Y}$ así las cosas, el conocimiento como objeto de educación exige la investigación de la educación, es decir, exige que la educación se convierta en objeto de conocimiento, bien como Pedagogía cognitiva 
o bien como Didáctica, respectivamente, pero, además de responder a por qué se produjo un determinado acontecimiento educativo y a cómo se puede lograr un determinado acontecimiento educativo, hay que responder, también, a cómo se justifica ese acontecimiento como acontecimiento educativo y esta es una cuestión que sólo se responde desde el conocimiento que tenemos del fenómeno educativo, en tanto que se construye con el significado de 'educación' desde la Pedagogía. Esa es la pregunta desde la Pedagogía, no por mejorar nuestro modo de conocer, ni por mejorar nuestro modo de enseñar, sino la pregunta por la educación misma desde conceptos con significación intrínseca al ámbito de conocimiento 'educación'. Conocer un área cultural no es enseñar, porque, como acabamos de ver, las competencias que se requieren en cada caso son distintas y enseñar no es educar, porque podemos afirmar que hay enseñanzas que no educan, con fundamento en el significado propio de esos términos. En consecuencia, la lógica de saber no es la lógica de hacer saber y esta no es la lógica de educar, necesariamente ${ }^{5}$.

El ámbito de educación, tal como se usa en este contexto de argumentación, no es un espacio físico, sino un concepto derivado de la valoración educativa del área de experiencia que utilizamos como instrumento y meta de educación. El ámbito de educación es resultado de la valoración educativa del área de experiencia que utilizamos para educar y por eso en el ámbito de educación se integran el significado de educación, los procesos de intervención, las dimensiones de intervención y las áreas de experiencia y las formas de expresión en cada acepción técnica de ámbito.

El ámbito de educación, que es siempre expresión del área cultural valorada como

5 Touriñán, J. M. (2018b). La significación del conocimiento de la educación y su capacidad de resolución de problemas: fundamentos desde el conocimiento pedagógico. Revista Boletín REDIPE, 7 (1), enero, 25-61. objeto e instrumento de educación, integra los siguientes componentes: área de experiencia con la que vamos a educar, formas de expresión convenientes para educar con esa área, criterios de significado de educación reflejados en rasgos de carácter y sentido inherentes al significado de educar, dimensiones generales de intervención que vamos a utilizar en la educación, procesos de educación que deben seguirse y acepción técnica de ámbito. Integrar estos componentes es lo que hace el conocimiento de la educación con cada área cultural para hablar con propiedad conceptual de educar "con" un área cultural como concepto distinto de enseñar un área cultural y conocer un área cultural que forma parte del currículo ${ }^{6}$.

Lo primero que alcanzamos en el conocimiento de una cosa, incluso sin darnos cuenta, es su aspecto, es su figura o tipo de cosa que es. El aspecto o idea, en el sentido más puro de la tradición griega, es el conjunto de rasgos fisonómicos o característicos de que una cosa es. Precisamente por eso el primer paso en nuestra experiencia de llegar a saber en sentido pleno es ser capaz de discernir. Saber es, en primer lugar, identificar y discernir y ese significado le da sentido a la frase "sé quién eres, pero no te conozco":

Además de discernir, identificando una cosa por su aspecto, tenemos que distinguir una cosa de otra; definimos, atendiendo a la circunscripción de sus rasgos con precisión. Por medio de estos rasgos abrimos el camino al conocimiento verdadero pleno de saber. Pero buscar el perfil unitario de rasgos tampoco es suficiente, de manera que tiene sentido afirmar que saber no es sólo discernir distinguiendo rasgos, saber es, en sentido pleno, entender?

6 Touriñán, J. M. y Longueira, S. (Coords.) (2018). La construcción de ámbitos de educación. Pedagogía general y aplicada. Santiago de Compostela: Andavira.

7

Touriñán, J. M. (2016). Pedagogía general. Principios de educación y principios de intervención. A Coruña: Bello y Martínez. 
El sentido propio de la definición real nos exige, además de discernir y distinguir rasgos, entender, o sea, nos exige saber en sentido pleno: 1) demostrar la necesidad lógica de unos caracteres o rasgos constitutivos, 2) razonar teórica y prácticamente sobre sus principios y 3) calibrar la impresión de realidad que nos trasmiten. $Y$ así las cosas, tiene sentido afirmar que una fuente constante de mala comprensión y de consideraciones erróneas es la indefinición del significado. Por la vaguedad del significado interpretamos mal a otras personas, a otras cosas, a nosotros mismos: por ambigüedad, distorsionamos y tergiversamos ${ }^{8}$.

De acuerdo con las distinciones realizadas, hablar de conocimiento de la educación es lo mismo que interrogarse acerca de la educación como objeto de conocimiento, lo que equivale a formularse una doble pregunta ${ }^{9}$.

- Qué es lo que hay que conocer para entender y dominar el ámbito de la educación; o lo que es lo mismo, cuales son los componentes del fenómeno educativo que hay que dominar para entender dicho fenómeno.

- Cómo se conoce ese campo; o dicho de otro modo, qué garantías de credibilidad tiene el conocimiento que podamos obtener acerca del campo de la educación.

Hay que asumir sin prejuicios que la Pedagogía es conocimiento de la educación y este se obtiene de diversas formas, pero, en última instancia, ese conocimiento, por principio de significación, sólo es válido si sirve para educar; es decir, para transformar la información en conocimiento y este en educación, desde conceptos con significación intrínseca, autóctona, al ámbito de estudio y análisis: la educación. Por una

8 Touriñán, J. M. (2018d). El lenguaje de la educación; más allá de lemas y metáforas. Revista Boletín Redipe, 7 (10), octubre, 31-58.

9 Touriñán, J. M. (2015). Pedagogía mesoaxiológica y concepto de educación. Santiago de Compostela: Andavira (Disponible la $2^{a}$ edición de 2016). parte, hay que saber en el sentido más amplio del término (sé qué, sé cómo y sé hacer); por otra parte, hay que enseñar (que implica otro tipo de saber distinto al de conocer las áreas de experiencia cultural); y, por si eso fuera poco, además, hay que educar, que implica, no sólo saber y enseñar, sino también dominar el carácter y sentido propios del significado de 'educación', para aplicarlo a cada área experiencia cultural con la que educamos. Cuando abordamos el área de experiencia cultural desde la mirada pedagógica, nuestra preocupación intelectual nos permite distinguir entre "saber Historia", "enseñar Historia" y "educar con la Historia", entendida esta como una materia de área cultural que forma parte del currículo junto con otras y se ha convertido desde la Pedagogía en ámbito de educación.

Para mí, queda claro que:

- Hablar de los conocimientos de la educación es lo mismo que hablar del conjunto de conocimientos teóricos, tecnológicos y prácticos que la investigación va consolidando acerca del ámbito de realidad que es la educación. Son en sí mismos conocimientos de un área cultural. Pero, en este caso, son el área cultural específica -la de la educación-, que se convierte en sí misma en objeto de conocimiento (educación como objeto de conocimiento). Hablamos en este caso de conocimiento de la educación que tendremos que utilizar para educar con un área cultural determinada, por ejemplo, el área cultural "Historia" o "Artes", etc.

- Hablar de los conocimientos de las áreas culturales es hablar de los conocimientos teóricos, tecnológicos y prácticos que los especialistas de cada área -matemáticos, físicos, psicólogos, médicos, técnicos del arte, etc.- han ido consolidando con sus investigaciones. Hablamos en este caso 
del conocimiento del área cultural que se va a convertir en objeto de educación y de enseñanza, según el caso.

- Hablar del conocimiento como objeto de educación es hablar de una determinada parcela del conocimiento de la educación, aquella que nos permite intervenir para mejorar nuestro modo de conocer. Hablamos en este caso de la posibilidad de mejorar nuestra capacidad de conocer, entendido este concepto en el sentido más amplio y aplicado a cada área cultural a enseñar.

Es fundamental distinguir entre "ámbito de conocimiento", conocimiento del ámbito", por un lado, y "ámbito de educación", por otro lado, para saber de qué hablamos en cada caso; o lo que es lo mismo, es fundamental distinguir entre Educación y Pedagogía, por un lado, y "ámbito de educación", por otro, pues este concepto nace de la valoración del área cultural como educativa, ajustándola al significado de educar que se obtiene con la Pedagogía ${ }^{10}$.

Si no confundimos conocimiento de áreas culturales y conocimiento de la educación, ni es verdad que el profesor es un aprendiz de las áreas culturales que enseña, ni es verdad que necesariamente el que más Arte sabe es el que mejor lo enseña, ni es verdad que el que mejor domine una destreza es el que mejor enseña a otro a dominarla, a menos que, tautológicamente, digamos que la destreza que domina es la de enseñar, ni es verdad que, cuando se enseña, estamos utilizando siempre el contenido cultural como instrumento de logro del carácter y sentido propio del significado de educación, porque enseñar no es educar. Es objetivo de la pedagogía transformar la información en conocimiento y el conocimiento en educación, construyendo ámbitos de

10 Touriñán, J. M. y Sáez, R. (2015). La mirada pedagógica. Teoría de la educación, metodología y focalizaciones. Santiago de Compostela: Andavira. educación desde las diversas áreas culturales, y precisamente por eso podemos decir que a la Pedagogía le corresponde valorar cada área cultural como educación y construirla como "ámbito de educación".

Esto es así, porque cada una de esas actividades requiere distintas competencias y destrezas para su dominio, y la práctica y perfección en una de ellas no genera automáticamente el dominio de la otra. En rigor lógico, hay que aceptar que el conocimiento de la educación es, pues, un conocimiento especializado que permite al pedagogo explicar, interpretar y decidir la intervención pedagógica adecuada al área cultural que es objeto de enseñanza y educación, según el caso.

La educación es actividad ${ }^{11}$. La educación se ajusta al criterio de finalidad; la actividad educativa es "educativa", porque tiene la finalidad de educar y ajusta el significado a los criterios de definición del término. En consonancia con lo que hemos argumentado en este prólogo, hay que afirmar que la finalidad de la educación, es, por definición, que el educando adquiera conocimientos, actitudes y destrezas-habilidades-hábitos que lo capacitan, desde cada actividad común interna (pensar, sentir afectivamente-tener sentimientos, querer, elegir-hacer (operar), decidir-actuar (proyectar) y crear: construir simbolizando) y externa (juego, trabajo, estudio, intervención, indagación y relación), para decidir y realizar sus proyectos, dando respuesta de acuerdo con las oportunidades a las exigencias que se plantean en cada situación ${ }^{12}$.

11 Touriñán, J. M. (2019). Estudiar es actividad común externa y siempre educamos con la actividad. Una aproximación desde la perspectiva mesoaxiológica. Teoría de la educación. Revista interuniversitaria, 31 (2), 7-31.

12 Touriñán, J. (2019b). La relación educativa es un concepto con significado propio que requiere concordancia entre valores y sentimientos en cada interacción. Sophia, colección de Filosofía de la Educación, 26 (1), 223-279. 
Educar es una tarea y un resultado. Y, desde la Pedagogía, educar es también un proceso que realiza el significado de la educación en cualquier ámbito educativo construido, desarrollando las dimensiones generales de intervención y las competencias adecuadas, las capacidades específicas y las disposiciones básicas de cada educando para el logro de conocimientos, actitudes y destrezas-habilidades-hábitos relativos a las finalidades de la educación y a los valores guía derivados de las mismas en cada actividad interna y externa del educando y, para ello, utiliza los medios internos y externos convenientes a cada actividad, de acuerdo con las oportunidades.

Esa es la competencia que hay que lograr, porque el sistema educativo está definido en las leyes para educar y no solo para enseñar. Estamos ante una alternativa que coloca en el primer plano a las finalidades orientadas a optimizar el significado de educación, que atiende a las finalidades intrínsecas y extrínsecas y que asume la orientación formativa temporal para la condición humana. $Y$ esto resalta la evidente necesidad en la formación de profesores de prepararlos para valorar educativamente cualquier medio que vayan a utilizar para educar. En definitiva, resalta la necesidad de capacitar a los profesores, en tanto que pedagogos, en la perspectiva mesoaxiológica, para construir ámbitos de educación con las áreas de experiencia cultural, generar el diseño educativo de la actuación y realizar intervención pedagógica correspondiente, sabiendo cuánto de educación común, de específica y de educación especializada hay en su intervención y cuánto de diseño educativo y de ámbito de educación están cumplimentando en cada intervención con el área de experiencia utilizada.

La Pedagogía mesoaxiológica transforma un contenido de área cultural en ámbito de educación; es comprensión del medio, valorándolo como educativo. La Pedagogía es mesoaxiológica porque equivale a comprender (logos) la valoración educativa (axio) del medio o instrumento utilizado (meso). Es importante mantener esta precisión e identificación como ámbito de educación, porque su defensa y fundamentación contribuye a formar criterio en los profesionales de la educación acerca del valor educativo de un área cultural y su posibilidad como conocimiento pedagógico. Tiene sentido afirmar, como ya hemos dicho, que no es lo mismo "saber Historia", "enseñar Historia" y "educar con la Historia"; sólo en el último caso la Historia se convierte en ámbito de educación y, por tanto, en instrumento y meta de la intervención pedagógica.

La Pedagogía mesoaxiológica es pedagogía mediada, relativa al medio $\mathrm{o}$ ámbito de educación que se construye para educar con un área cultural. La Pedagogía Mesoaxiológica es pedagogía del medio o ámbito de educación, porque cada área cultural tiene que ser valorada como educación y construida como "ámbito de educación", un concepto que tiene significación propia, autóctona, desde el conocimiento de la educación y está vinculado a los rasgos de carácter y sentido inherentes al significado de educación, a los procesos de intervención, a las dimensiones generales de intervención y a las áreas de experiencia y a las formas de expresión que mejor se ajustan a cada área en cada acepción técnica de ámbito. En relación con el medio o ámbito de educación que se construye en cada caso con un área de experiencia cultural, la Pedagogía es Pedagogía Mesoaxiológica,

En mi opinión, la Pedagogía es Mesoaxiológica: educamos $\mathrm{CON}$ las áreas culturales $\mathrm{y}$, con fundamento en la decisión técnica, que es la que me permite valorar el área cultural como educativa, construyo el ámbito de educación común, específico y especializado que corresponda, genero el diseño educativo apropiado y realizo la intervención pedagógica 
requerida en forma de acción concreta, controlada y programada.

En perspectiva mesoaxiológica, la posibilidad de acción pedagógica es definida: además de enseñar, hay que educar, transformando información en conocimiento y éste, a su vez, en educación. $Y$ hay además un riesgo constante: el conocimiento de la educación fundamenta la decisión pedagógica, que es técnica y de perspectiva mesoaxiológica, pero no es suficiente para garantizar que nadie saltará el limite, politizando la educación, adoctrinando, manipulando o eligiendo en lugar del educando su sentido de acción y su modo de vida.

En resumen, en perspectiva mesoaxiológica, transformar información en conocimiento y este conocimiento en educación exige entender la relación que hay entre el conocimiento de la educación y el concepto de educación, a fin de adquirir competencia para construir el ámbito de educación, hacer el diseño educativo y generar la intervención, de manera que la función de educar sea vista también como competencia técnica que se ejerce en cada caso con mentalidad pedagógica específica y con mirada pedagógica especializada. La perspectiva mesoaxiológica se resume, conceptualmente hablando, en los siguientes postulados ${ }^{13}$ :

1. Conocer, enseñar y educar tienen distinto significado; el conocimiento de áreas culturales no es el conocimiento de la educación. El conocimiento de la educación fundamenta el nexo entre mentalidad pedagógica específica mirada pedagógica especializada y acción educativa concreta controlada y programada para formar la condición individual social histórica y de especie de cada educando

2. Transformamos información en conocimiento y conocimiento en educación, ajustándolo al significado de educación,

13 http://dondestalaeducacion.com/conceptos/40-laperspectiva-mesoaxiologica-de-la-pedagogia.html utilizando la actividad común del educando para intervenir y buscando la concordancia entre valores y sentimientos en el paso del conocimiento a la acción

3. La función pedagógica es técnica, no política, aunque la educación sea un asunto de interés político, igual que la salud o la vivienda; la decisión en Pedagogía, que es conocimiento de la educación, es tecnoaxiológica y mesoaxiológica. Decidimos con fundamento en el conocimiento verdadero del ámbito en el que se trabaja

4. En perspectiva mesoaxiológica construimos ámbitos de educación hacemos el diseño educativo pertinente y generamos la intervención pedagógica atendiendo a principios de educación y a principios de intervención que se justifican con el conocimiento de la educación

5. Usamos la actividad común de manera controlada para lograr actividad educada y educar la actividad con mentalidad pedagógica específica y mirada pedagógica especializada desde los elementos estructurales de la intervención, porque sin la actividad común es imposible educar y gracias a ella se hace posible que el educando sea agente actor y cada vez mejor agente autor de su propios proyectos y actos.

Estoy convencido de que, como carrera, el futuro de la Pedagogía está vinculado a la necesidad social de calidad de la educación. La calidad de educación es una necesidad social y se configura como un objetivo posible y necesario. La respuesta educativa afecta a la realidad interna y externa del educando en cada circunstancia y de acuerdo con las oportunidades, y dado que cualquier respuesta no es igualmente valiosa ni ajustada al significado de educación, inexorablemente tenemos que hacer frente a 
problemas de valorar la calidad en cada acción educativa. $Y$ eso quiere decir que el futuro de la Pedagogía depende de que esa relación se haga constante y patrimonial por parte de los pedagogos $^{14}$.

También estoy convencido de que, como disciplina, la Pedagogía del futuro es la pedagogía aplicada y por medio de su consistencia epistemológica le damos a la Pedagogía el lugar que le corresponde y preservamos su relación con la calidad de la educación, pues hacemos que sirva para educar reforzando su capacidad de resolución de problemas. Y esto que deuda escrito en las páginas anteriores contribuye a profundizar en la pedagogía aplicada desde la perspectiva de hacer de la enseñanza un proceso educativo y no solo instructivo, porque nos compromete a enseñar educando, si nuestro objetivo es cumplir las finalidades de la educación.

\section{REFERENCIAS WEB PARA SACAR PARTIDO AL TEXTO}

Además de los libros citados a pie de página, el contenido sobre el concepto de educar se puede seguir profundizando por medio de la consulta de los siguientes documentos:

1. LÍNEA DE INVESTIGACIÓN: PEDAGOGÍA MESOAXIOLÓGICAY CONSTRUCCIÓN DE ÁMBITOS DE EDUCACIÓN PROYECTO CONSTRUCCIÓN DE ÁMBITOS DE EDUCACIÓN "EDUCERE AREA: EDUCAR CON EL ÁREA CULTURAL”,

Este documento da acceso a los cuestionarios de perspectiva mesoaxiológica

disponible en http://dondestalaeducacion.com/ files/6315/7963/1664/Proy Educere Area-Lin Invest PMyCAE.pdf

14 Touriñán, J. M. (2020). Función pedagógica, competencia técnica y transferencia de conocimiento. Colombia-Nueva York: Redipe (Bowker-Books).
2. ARTÍCULO DE AMPLIACIÓN DE CONTENIDO, PUBLICADO EN RBR 6(12)2017, PP. 24-65: "EL CONCEPTO DE EDUCACIÓN: CARÁCTER, SENTIDO PEDAGÓGICO, SIGNIFICADO Y ORIENTACIÓN FORMATIVA TEMPORAL. HACIA LA CONSTRUCCIÓN DE ÁMBITOS DE EDUCACIÓN"

disponible en http://dondestalaeducacion. com/files/3715/4496/1681/ ReBolRedip_6_12_24-65_Dic17_Art. Concept_Educ.pdf

Este artículo está resumido en un MICROVIDEO del mismo título

disponible en https://youtu.be/UwOE--fPbQI

3. ADEMÁS SE COMPLETA EL CONTENIDO DE LA CONFERENCIA CON UN VIDEO EN YOUTUBE DE POWERPOINT SOBRE INTERVENCIÓN PEDAGÓGICA COMO PROBLEMA DE TODOS EN LA EDUCACIÓN: "CLAVES PARA COMPRENDER LA INTERVENCIÓN PEDAGÓGICA EN LA ESCUELA: LA INTERVENCIÓN EDUCATIVA ES UN PROBLEMA DE TODOS".

disponible en https://youtu.be/BcCaS2Pv-UE

4. EL CONTENIDO SOBRE EL CONCEPTO SE PUEDE SEGUIR PROFUNDIZANDO POR MEDIO DE LA CONSULTA DE LOS DOCUMENTOS DE CONCEPTO QUE ESTÁN EN LA PÁGINA WEB HTTP://DONDESTALAEDUCACION. COM/RELATIVOS A CONCEPTO DE EDUCACIÓN Y PERSPECTIVA MESOAXIOLÓGICA:

\section{EL SIGNIFICADO DE 'EDUCACIÓN' COMO CONFLUENCIA DE CRITERIOS DE DEFINICIÓN}

http://dondestalaeducacion.com/conceptos/ generica $17 . \mathrm{html}$ 
2. EL CONCEPTO DE 'EDUCACIÓN' COMO CONFLUENCIA DE SIGNIFICADOY ORIENTACIÓN FORMATIVA TEMPORAL

http://dondestalaeducacion.com/conceptos/ generica_18.html

3. PRINCIPIOS DE EDUCACIÓN Y PRINCIPIOS DE INTERVENCIÓN PEDAGÓGICA

http://dondestalaeducacion.com/conceptos/ generica 19.html

4. CARÁCTER QUE DETERMINA EL SIGNIFICADO DE 'EDUCACIÓN'

http://dondestalaeducacion.com/conceptos/ generica4.html

5. SENTIDO DE LA EDUCACIÓN QUE CUALIFICA SU SIGNIFICADO

http://dondestalaeducacion.com/conceptos/ generica_24.html

6. CONCEPTO DE EDUCACIÓN Y PEDAGOGÍA MESOAXIOLÓGICA

http://dondestalaeducacion.com/conceptos/ generica_25.html

7. LAPERSPECTIVAMESOAXIOLÓGICA DE LA PEDAGOGÍA

http://dondestalaeducacion.com/conceptos/40la-perspectiva-mesoaxiologica-de-lapedagogia.html

5. PARA AMPLIAR INFORMACIÓN, SE CONSULTARÁN LAS DIRECCIONES DE ENLACES:

- Videos disponibles en el canal YouTube DONDESTALAEDUCACION https://www.youtube.com/channel/ UCzbNAzwFApNMViix5 HDzOW

https://docs.google.com/viewerng/ viewer?url=http://dondestalaeducacion.com/ files/2015/8654/4580/videos_disponibles youtube 10abr20.pdf

- $\quad$ Conceptos de la web dondestalaeducacion, http://dondestalaeducacion.com/conceptos.html 